ESAIM: PROCEEDINGS, September 2007, Vol.21, 45-64

Gabriel Caloz \& Monique Dauge, Editors

\title{
FINITE ELEMENT METHOD WITH PATCHES FOR POISSON PROBLEMS IN POLYGONAL DOMAINS
}

\author{
Alexei Lozinski ${ }^{1}$, JACQUes Rappaz ${ }^{2}$ AND JoËL WAgneR ${ }^{3}$ \\ Dedicated to Prof. Michel Crouzeix
}

\begin{abstract}
In this paper we consider the finite element approximation of the singularities of the solution of Poisson problems in a polygonal domain with reentrant corners or changing DirichletNeumann boundary conditions. We use a correction algorithm with patches of elements to improve the a priori error estimates and to obtain the same order as the optimal estimate when everything is regular. We give an application of the correction method to the problem of glacier modeling.
\end{abstract}

\section{INTRODUCTION}

The aim of this paper is to use the finite element method with patches, introduced by Glowinski et al. in $[11,12]$, in the setting of certain problems with singular solutions as studied for example by Raugel [20] and Grisvard [15,16], earlier by Babuska [1], and after by Dauge [8], Brenner [4], Cai et al. [5]. The results of this paper have first been presented by Wagner in [22] but we give here some simpler and more general proofs for them.

A method for the numerical solution of elliptic problems with multi-scale data using multiple levels of not necessarily nested grids is introduced in [12] and analyzed in [11]. We recall this algorithm here for the convenience of the reader. The method consists in calculating successive corrections to the solution in patches whose shape is not necessarily conforming with the global coarse grid. We consider a polygonal domain $\Omega \subset \mathbb{R}^{2}$ with boundary $\partial \Omega$ split into two parts $\Gamma_{1}, \Gamma_{2}$, and $V=\left\{v \in H^{1}(\Omega)\right.$ such that $v=0$ on $\left.\Gamma_{1}\right\}$ where $H^{1}(\Omega)$ denotes the usual Sobolev space of functions with first derivatives in $L^{2}(\Omega)$. Let $a: V \times V \rightarrow \mathbb{R}$ be a bilinear, symmetric, continuous and coercive form. If $f \in L^{2}(\Omega)$, we are looking for the unique $u \in V$ such that

$$
a(u, v)=\int_{\Omega} f v \mathrm{~d} x, \quad \forall v \in V .
$$

An approximation of $u$ by the finite element method of order $r$ consists in introducing a regular family of triangulations $\mathcal{T}_{H}$ of $\bar{\Omega}[6$, Sect. 17], defining

$$
\begin{aligned}
V_{H}= & \left\{g: \bar{\Omega} \rightarrow \mathbb{R} \text { continuous such that }\left.g\right|_{K} \in \mathbb{P}_{r}(K), \forall K \in \mathcal{T}_{H}\right. \\
& \text { and } \left.g=0 \text { on } \Gamma_{1}\right\},
\end{aligned}
$$

\footnotetext{
1 Dept. of Mathematics, University of Houston, 4800 Calhoun Road, Houston, Texas 77204-3008, USA (alozinski@gmail.com).

2 Section of Mathematics, Ecole Polytechnique Fédérale de Lausanne (EPFL), 1015 Lausanne, Switzerland (jacques.rappaz@epf 1. ch).

3 mail@joel.wagner.name.

(C) EDP Sciences, SMAI 2007
} 
where $\mathbb{P}_{r}(K)$ is the space of polynomials of degree $\leq r$ on triangle $K \in \mathcal{T}_{H}$, and calculating $u_{H} \in V_{H}$ satisfying $a\left(u_{H}, v\right)=\int_{\Omega} f v \mathrm{~d} x, \forall v \in V_{H}$. Consider now $\Lambda \subset \Omega$ another open polygonal domain wherein we would like to obtain a better precision on the solution $u$ than the one given by $u_{H}$. Take note that $\bar{\Lambda}$ is not necessarily the union of several triangles $K$ of $\mathcal{T}_{H}$. Let $\mathcal{T}_{h}$ be a regular family of triangulations of $\bar{\Lambda}$ and consider

$$
\begin{aligned}
V_{h}= & \left\{g: \bar{\Omega} \rightarrow \mathbb{R} \text { continuous such that }\left.g\right|_{K} \in \mathbb{P}_{s}(K), \forall K \in \mathcal{T}_{h}\right. \\
& \text { and } \left.g=0 \text { on } \Omega \backslash \Lambda, g=0 \text { on } \Gamma_{1}\right\} .
\end{aligned}
$$

We call $H=\max _{K \in \mathcal{T}_{H}} \operatorname{diam}(K)$ and $h=\max _{K \in \mathcal{T}_{h}} \operatorname{diam}(K)$. Setting $V_{H h}=V_{H}+V_{h}$ as approximation for $u$ we are looking for the function $u_{H h} \in V_{H h}$ satisfying

$$
a\left(u_{H h}, v\right)=\int_{\Omega} f v \mathrm{~d} x, \quad \forall v \in V_{H h} .
$$

Remark that, in general, $V_{H h}$ is not a direct sum of $V_{H}$ and $V_{h}$. In practice, it is not possible to determine a finite element basis of $V_{H h}$ and hence to compute directly $u_{H h}$. It is the reason why we use the following algorithm for computing $u_{H h}$ : At iteration $n=0$ we initialize with $u_{H}^{0}=u_{H}, u_{h}^{0}=0$ and choose a relaxation parameter $\omega \in(0 ; 2)$. Then for $n=1,2,3, \ldots$ we write $u^{n-1}=u_{H}^{n-1}+u_{h}^{n-1}$ with $u_{H}^{n-1} \in V_{H}, u_{h}^{n-1} \in V_{h}$. We write the first step of the $n$-th iteration of the algorithm as follows:

Find $v_{h} \in V_{h}$ s.t. $a\left(v_{h}, \varphi\right)=\int_{\Omega} f \varphi \mathrm{d} x-a\left(u_{H}^{n-1}, \varphi\right), \quad \forall \varphi \in V_{h}$.

Set $u_{h}^{n}=(1-\omega) u_{h}^{n-1}+\omega v_{h}$.

The same holds for the second step which writes out explicitly:

Find $v_{H} \in V_{H}$ s.t. $a\left(v_{H}, \varphi\right)=\int_{\Omega} f \varphi \mathrm{d} x-a\left(u_{h}^{n}, \varphi\right), \quad \forall \varphi \in V_{H}$.

Set $u_{H}^{n}=(1-\omega) u_{H}^{n-1}+\omega v_{H}$.

Remark that $u_{h}^{n}=u_{h}^{n-1}+\omega w_{h}$ where $w_{h} \in V_{h}$ is such that

$$
a\left(w_{h}, \varphi\right)=\int_{\Omega} f \varphi \mathrm{d} x-a\left(u_{H}^{n-1}+u_{h}^{n-1}, \varphi\right), \quad \forall \varphi \in V_{h},
$$

and that $u_{H}^{n}=u_{H}^{n-1}+\omega w_{H}$ where $w_{H} \in V_{H}$ is such that

$$
a\left(w_{H}, \varphi\right)=\int_{\Omega} f \varphi \mathrm{d} x-a\left(u_{H}^{n-1}+u_{h}^{n}, \varphi\right), \quad \forall \varphi \in V_{H} .
$$

In [11] it is proven that $u^{n}$ converges to $u_{H h}$ when $n$ tends to infinity and, provided $u \in H^{q}(\Omega)$ with $q=$ $\max (r, s)+1$, the approximation $u_{H h}$ to $u$ satisfies the a priori error estimate

$$
\left\|u-u_{H h}\right\| \leq C\left(H^{r}\|u\|_{H^{q}(\Omega \backslash \bar{\Lambda})}+h^{s}\|u\|_{H^{q}(\Lambda)}\right),
$$

where $C$ is a constant independent of $H$ and $h$, and $\|v\|=a(v, v)^{1 / 2}, \forall v \in V$.

At this point, taking inspiration from the described correction method we consider regularity and convergence results for problems with singularities due to changing Dirichlet-Neumann boundary conditions and domains with reentrant corners. Such problems have been studied for example by Raugel [20] and Grisvard [15, 16].

In the following, we discuss how patches can improve the quality of the solution and the convergence order in the grid size $H$ of the above described method on model problems. We consider problems where $u$ is not in $H^{2}(\Omega)$ and in principle we use polynomials of degree one $(r=s=1)$. In Section 1 we introduce some interpolation estimates used for the a priori error analysis to follow. In Section 2, we consider a Laplace problem with changing Dirichlet-Neumann boundary conditions. First we recall the regularity of solutions to this type of problems and present a priori error estimates. We improve the latter through the use of the correction algorithm with chosen patches (Proposition 2.1). In Section 3, following the same structure then above, we use the method on a Poisson-Dirichlet problem on a polygonal domain with reentrant corner. Finally, in Section 4 we apply the correction method to the problem of glacier modeling. We show how applying patches improves the precision of the computation of the stress field in the ice mass. 


\section{Preliminary Results}

In this section we introduce an estimate (Proposition 1.3) for the finite element interpolation on two superimposed but not necessarily nested grids in a two-dimensional polygonal domain $\Omega$ of the function

$$
\varphi=r^{\gamma} g(\theta)
$$

where $r, \theta$ are the polar coordinates, $0<\gamma<1$ is a number and $g(\theta) \in C^{\infty}([0,2 \pi])$ is a given function. We suppose from now on that $\Omega$ does not contain the ray $\theta=0$ but $\bar{\Omega}$ contains the origin, hence the function $\varphi$ does not belong to $H^{2}(\Omega)$ and we cannot expect to find a finite element approximation of $\varphi$ which is order 1 accurate in $H^{1}$ norm on a general grid.

Let $C_{a}$ denote the intersection of $\Omega$ with the open disc of radius $a>0$ centered at 0 . We have for any $a \in(0, R)$ where $R$ is the radius of the smallest disc centered at the origin that contains $\Omega$ :

$$
|\varphi|_{H^{2}\left(\Omega \backslash \bar{C}_{a}\right)}^{2} \leq C \int_{0}^{2 \pi} \int_{a}^{R} r^{2 \gamma-4}\left(g(\theta)^{2}+g^{\prime}(\theta)^{2}+g^{\prime \prime}(\theta)^{2}\right) r d r d \theta \leq \frac{C}{a^{2-2 \gamma}}\|g\|_{H^{2}([0,2 \pi])}^{2}
$$

hence

$$
|\varphi|_{H^{2}\left(\Omega \backslash \bar{C}_{a}\right)} \leq \frac{C}{a^{1-\gamma}}\|g\|_{H^{2}([0,2 \pi])}
$$

with constant $C$ depending only on $\gamma$. By a similar calculation, we have

$$
|\varphi|_{H^{1}\left(C_{a}\right)} \leq C a^{\gamma}\|g\|_{H^{1}([0,2 \pi])}
$$

Lemma 1.1. For the setting above, function $\varphi$ given by (4) and any $a \in(0, R)$, there exists a function $\tilde{\varphi} \in$ $H^{2}(\Omega)$ such that $\left.\tilde{\varphi}\right|_{\Omega \backslash \bar{C}_{a}}=\left.\varphi\right|_{\Omega \backslash \bar{C}_{a}}$ and

$$
\begin{aligned}
|\tilde{\varphi}|_{H^{2}(\Omega)} & \leq \frac{C}{a^{1-\gamma}}\|g\|_{H^{2}([0,2 \pi])} \\
|\tilde{\varphi}|_{H^{1}\left(C_{a}\right)} & \leq C a^{\gamma}\|g\|_{H^{1}([0,2 \pi])}
\end{aligned}
$$

with a constant $C$ depending only on $\gamma$. If $g\left(\theta_{0}\right)=0$ for some $\theta_{0}$ then $\tilde{\varphi}$ can be chosen so that it vanishes on the $\operatorname{ray} \theta=\theta_{0}$.

Proof. Let us pick a function $\chi \in C^{2}([0,1])$ such that

$$
\chi(0)=0, \chi^{\prime}(0)=0, \chi(1)=1, \chi^{\prime}(1)=\gamma
$$

and define $\tilde{\varphi}$ by

$$
\tilde{\varphi}=\left\{\begin{array}{l}
\varphi \text { in } \Omega \backslash C_{a} \\
a^{\gamma} \chi\left(\frac{r}{a}\right) g(\theta) \text { in } \bar{C}_{a}
\end{array}\right.
$$

We have then $\tilde{\varphi} \in H^{2}(\Omega)$ and $|\tilde{\varphi}|_{H^{2}\left(\Omega \backslash \bar{C}_{a}\right)} \leq C a^{\gamma-1}\|g\|_{H^{2}([0,2 \pi])}$ by $(5)$. By change of variables $r \rightarrow r / a$, it is easy to see that the analogous estimate holds inside $C_{a}$, namely $|\tilde{\varphi}|_{H^{2}\left(C_{a}\right)} \leq C a^{\gamma-1}|| g \|_{H^{2}([0,2 \pi])}$, hence (7). The estimate (8) follows in the same manner.

We introduce $\mathcal{T}_{H}$ a regular family of triangulations (see Ciarlet [6, Sect. 17]) with triangles $K$ over $\bar{\Omega}$ and call $H=\max _{K \in \mathcal{T}_{H}} \operatorname{diam}(K)$. We suppose that $\mathbf{0}$ is a node of $\mathcal{T}_{H}$. Let $r_{H}$ be the standard nodal interpolant by piecewise linear functions on $\mathcal{T}_{H}$. Let moreover $\Gamma$ be a (possibly empty) part of $\partial \Omega$ consisting of several straight segments of non zero length starting from the origin and suppose that $\varphi$ of (4) vanishes on $\Gamma$. Consider the standard finite element space

$$
\begin{aligned}
V_{H}= & \left\{g \in C^{0}(\bar{\Omega}) \text { such that }\left.g\right|_{K} \in \mathbb{P}_{1}(K), \forall K \in \mathcal{T}_{H}\right. \\
& \text { and } g=0 \text { on } \Gamma\}
\end{aligned}
$$


We recall the following result [3, Theorem 6.3], which we prove here for completeness of exposition.

Lemma 1.2. There exists an element $\varphi_{H} \in V_{H}$ such that

$$
\left|\varphi-\varphi_{H}\right|_{H^{1}(\Omega)} \leq C H^{\gamma}\|g\|_{H^{2}([0,2 \pi])}
$$

where $\varphi$ is defined by (4) and $C$ is a constant depending only on $\gamma$ and the regularity parameter of $\mathcal{T}_{H}$.

Proof. Take $\varphi_{H}=r_{H} \tilde{\varphi}$ where $\tilde{\varphi}$ is the function constructed in Lemma 1.1 for $a=H$. Note that $\left.\tilde{\varphi}\right|_{\Gamma}=0$ and therefore $\varphi_{H} \in V_{H}$. Indeed, each segment from $\Gamma$ lies on the ray $\theta=\theta_{0}$ for some $\theta_{0}$. The function $\varphi$ can vanish on such a segment only if $g\left(\theta_{0}\right)=0$, meaning that $\tilde{\varphi}$ vanishes on this segment as well. We estimate now

$$
\left|\tilde{\varphi}-\varphi_{H}\right|_{H^{1}(\Omega)} \leq C H|\tilde{\varphi}|_{H^{2}(\Omega)} \leq C H^{\gamma}\|g\|_{H^{2}([0,2 \pi])}
$$

by standard approximation results from [6] and (7). Furthermore,

$$
\left|\varphi-\varphi_{H}\right|_{H^{1}(\Omega)} \leq\left|\tilde{\varphi}-\varphi_{H}\right|_{H^{1}(\Omega)}+|\varphi|_{H^{1}\left(C_{H}\right)}+|\tilde{\varphi}|_{H^{1}\left(C_{H}\right)} \leq C H^{\gamma}\|g\|_{H^{2}([0,2 \pi])}
$$

by (6) and (8). Hence (10) is recovered.

Let us now consider a family of domains $\Lambda_{\epsilon} \subset \Omega$ such that $C_{\epsilon} \subset \Lambda_{\epsilon}$ and introduce regular triangulations $\mathcal{T}_{h}$ on $\bar{\Lambda}_{\epsilon}$ with $h=\max _{K \in \mathcal{T}_{h}} \operatorname{diam}(K) \leq \epsilon$. As before, we suppose that $\mathbf{0}$ is a node of $\mathcal{T}_{h}$ and let $r_{h}$ be the standard nodal interpolant by piecewise linear functions on $\mathcal{T}_{h}$. Introduce the standard finite element space on $\mathcal{T}_{h}$ :

$$
\begin{aligned}
V_{h}= & \left\{g \in C^{0}(\bar{\Omega}) \text { such that }\left.g\right|_{K} \in \mathbb{P}_{1}(K), \forall K \in \mathcal{T}_{h}\right. \\
& \text { and } \left.g=0 \text { on } \Omega \backslash \Lambda_{\epsilon} \text { and on } \Gamma\right\},
\end{aligned}
$$

Proposition 1.3. There exists an element $\varphi_{H h} \in V_{H}+V_{h}$ such that

$$
\left|\varphi-\varphi_{H h}\right|_{H^{1}(\Omega)} \leq C\left(\frac{H}{\epsilon^{1-\gamma}}+h^{\gamma}\right)\|g\|_{H^{2}([0,2 \pi])}
$$

where $\varphi$ is defined by (4) and $C$ is a constant independent of $H, h, \epsilon$ and $g$.

Proof. Let $\tilde{\varphi}$ be the function constructed in Lemma 1.1 for $a=\epsilon$. We take $\varphi_{H}=r_{H} \tilde{\varphi}$ and assure ourselves that $\varphi_{H} \in V_{H}$ as in the proof of Lemma 1.2. Let us now apply Lemma 1.2 on $\mathcal{T}_{h}$ instead of $\mathcal{T}_{H}$ to construct $\varphi_{h} \in W_{h}=\left\{g \in C^{0}\left(\bar{\Lambda}_{\epsilon}\right):\left.g\right|_{K} \in \mathbb{P}_{1}(K), \forall K \in \mathcal{T}_{h}\right.$ and $g=0$ on $\left.\Gamma\right\}$, for which we have

$$
\left|\varphi-\varphi_{h}\right|_{H^{1}\left(\Lambda_{\epsilon}\right)} \leq C h^{\gamma}\|g\|_{H^{2}([0,2 \pi])}
$$

It is easy to see that $r_{h} \tilde{\varphi} \in W_{h}$ and, by construction, $\left.\varphi_{h}\right|_{\partial \Lambda_{\epsilon} \cap \Omega}=\left.r_{h} \varphi\right|_{\partial \Lambda_{\epsilon} \cap \Omega}=\left.r_{h} \tilde{\varphi}\right|_{\partial \Lambda_{\epsilon} \cap \Omega}$. Consequently, we can take $\psi_{h} \in V_{h}$ such that $\left.\psi_{h}\right|_{\Lambda_{\epsilon}}=\varphi_{h}-r_{h} \tilde{\varphi}$ and set $\varphi_{H h}=\varphi_{H}+\psi_{h}$. We estimate then

$$
\left|\varphi-\varphi_{H h}\right|_{H^{1}(\Omega)} \leq\left|\tilde{\varphi}-\varphi_{H}\right|_{H^{1}(\Omega)}+\left|\varphi-\tilde{\varphi}-\psi_{h}\right|_{H^{1}\left(\Lambda_{\epsilon}\right)}
$$

But

$$
\left|\tilde{\varphi}-\varphi_{H}\right|_{H^{1}(\Omega)} \leq C H|\tilde{\varphi}|_{H^{2}(\Omega)} \leq C \frac{H}{\epsilon^{1-\gamma}}\|g\|_{H^{2}([0,2 \pi])}
$$

by standard approximation results from [6] and (7). Furthermore,

$$
\begin{aligned}
\left|\varphi-\tilde{\varphi}-\psi_{h}\right|_{H^{1}\left(\Lambda_{\epsilon}\right)} & \leq\left|\varphi-\varphi_{h}\right|_{H^{1}\left(\Lambda_{\epsilon}\right)}+\left|\tilde{\varphi}-r_{h} \tilde{\varphi}\right|_{H^{1}\left(\Lambda_{\epsilon}\right)} \\
& \leq\left. C h^{\gamma}|| g\right|_{H^{2}([0,2 \pi])}+C h|\tilde{\varphi}|_{H^{2}(\Omega)} \leq C\left(h^{\gamma}+\frac{h}{\epsilon^{1-\gamma}}\right)\|g\|_{H^{2}([0,2 \pi])}
\end{aligned}
$$

by (13) and (7). Noting that $h / \epsilon^{1-\gamma} \leq h^{\gamma}$ concludes the proof. 


\section{Problem With Change in BOUNDARY CONDITIONS}

The objective of this discussion is to use the correction algorithm described in the Introduction on a Poisson problem with changing Dirichlet-Neumann boundary conditions. We first recall the regularity of the solution to such a problem and give the a priori convergence results. Next we implement the problem numerically, and after comparing the theoretical orders with the ones we obtain numerically, we use different types of patches in order to improve the convergence order.

\subsection{Regularity result.}

Before introducing a model problem, see (19), we recall the analysis of the singular behavior as presented by Grisvard in [15, Section 4.4] and [16, Pages 49-51 and Section 2.4] through the following situation.

Consider the domain $\Omega_{\infty}=(-\infty ;+\infty) \times(0 ;+\infty)$. In all the following we set $H_{\text {loc }}^{m}\left(\bar{\Omega}_{\infty}\right)=\left\{f: \Omega_{\infty} \rightarrow\right.$ $\mathbb{R}$ such that $f \in H^{m}(\stackrel{\circ}{\omega})$ for any compact set $\omega \subset \bar{\Omega}_{\infty}, \stackrel{\circ}{\omega} \neq \varnothing$ its interior $\}$. With this notation we consider the problem of finding the functions $v \in H_{\mathrm{loc}}^{1}\left(\bar{\Omega}_{\infty}\right)$ verifying $\Delta v=0$ in $\Omega_{\infty}$ and obeying the following set of boundary conditions:

$$
\begin{array}{ll}
\frac{\partial v}{\partial n}=0 & \text { on }(-\infty ; 0) \times\{0\}, \text { and } \\
v=0 & \text { on }(0 ;+\infty) \times\{0\} .
\end{array}
$$

We denote $x_{1}$ and $x_{2}$ the two space variables and $(r, \theta)$ are the polar coordinates. The situation as well as the notation used are illustrated in Figure 1.

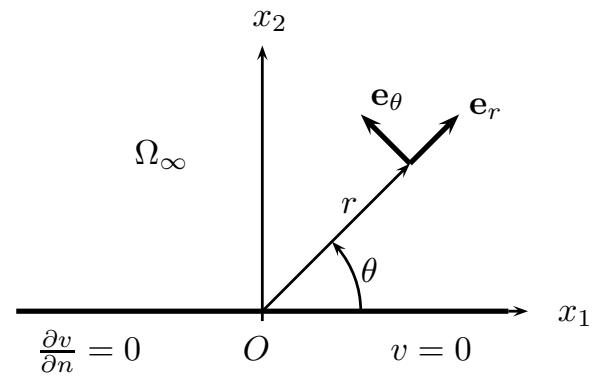

FIGURE 1. Illustration of the situation and notation.

To analyze these solutions, we consider the function $v=v(r, \theta)$ in polar coordinates. Taking into account the boundary conditions, we write

$$
v(r, \theta)=\sum_{m \geq 0} \rho_{m}(r) \sin \left(\frac{2 m+1}{2} \theta\right)
$$

and calculate its gradient and Laplacian. If we assume $\rho_{m}(r)=c_{m} r^{\gamma}$, the harmonic functions in $\Omega_{\infty}$ with boundary conditions (14) are expressed in polar coordinates by

$$
v(r, \theta)=\sum_{m \geq 0}\left(c_{m} r^{(2 m+1) / 2}+c_{-m} r^{-(2 m+1) / 2}\right) \sin \left(\frac{2 m+1}{2} \theta\right),
$$

where $c_{m}, c_{-m}$ are real coefficients. Note that $c_{0}$ and $c_{-0}$ are different a priori. Using the general expression (16) in polar coordinates, we analyze the regularity of these solutions. For $|\nabla v|^{2}$ to be locally integrable in $\bar{\Omega}_{\infty}$ a priori, we need to impose that, if $c_{m} \neq 0: \frac{2 m-1}{2} 2+1>-1$, and if $c_{-m} \neq 0:-\frac{2 m+3}{2} 2+1>-1$. The first condition is always verified for $m \geq 0$. The second implies $m<0$, and hence $c_{-0}=c_{-1}=c_{-2}=\ldots=0$. Thus, 


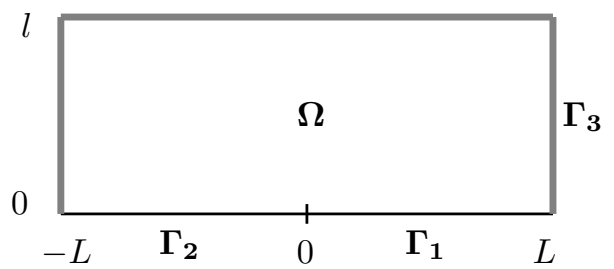

FIgURE 2. Illustration of the domain $\Omega$ and its boundaries $\Gamma_{i}, i=1,2,3$.

the functions of the form (16) that are $H_{\mathrm{loc}}^{1}\left(\bar{\Omega}_{\infty}\right)$ are expressed by

$$
v(r, \theta)=\sum_{m \geq 0} c_{m} r^{(2 m+1) / 2} \sin \left(\frac{2 m+1}{2} \theta\right) .
$$

Furthermore, considering the second derivatives of $v$, we note that, if $c_{0} \neq 0$, then $v$ does not belong to $H_{\mathrm{loc}}^{2}\left(\bar{\Omega}_{\infty}\right)$.

Thus we are interested in calculating $p$ such that $v$ of the form (17) with $c_{0} \neq 0$ is in $W_{\text {loc }}^{2, p}\left(\bar{\Omega}_{\infty}\right)$. For finding such $p$, we evaluate the second derivative of $r^{1 / 2} \sin (\theta / 2)\left(c_{0} \neq 0\right)$ and require it to be $p$-integrable with measure $r \mathrm{~d} r \mathrm{~d} \theta$. We obtain the relation $-\frac{3}{2} p+1>-1$, and hence $p<\frac{4}{3}$.

In conclusion, if $v \in H_{\text {loc }}^{1}\left(\bar{\Omega}_{\infty}\right)$ is an harmonic function in $\Omega_{\infty}$ verifying the boundary conditions (14), then $v \in W_{\text {loc }}^{2, p}\left(\bar{\Omega}_{\infty}\right)$ with $p \in\left[1 ; \frac{4}{3}\right)$. We denote by $\varphi(r, \theta)$ the component $m=0$ of $v$,

$$
\varphi(r, \theta)=c_{0} \sqrt{r} \sin (\theta / 2) .
$$

\subsection{Model problem and a priori error estimate.}

Let now $\Omega=(-L ; L) \times(0 ; l) \subset \mathbb{R}^{2}$ be a rectangular domain (see Figure 2$)$. We consider the following Poisson problem with homogeneous Dirichlet-Neumann boundary conditions:

For given $f \in L^{2}(\Omega)$, find $u \in H^{1}(\Omega)$ such that

$$
\begin{cases}-\Delta u=f & \text { in } \Omega, \\ u=0 & \text { on } \Gamma_{1}=\left\{\left(x_{1}, 0\right): 0<x_{1}<L\right\}, \\ \frac{\partial u}{\partial n}=0 & \text { on } \Gamma_{2}=\left\{\left(x_{1}, 0\right):-L<x_{1}<0\right\} \\ u=0 & \text { on } \Gamma_{3}=\partial \Omega \backslash\left(\Gamma_{1} \cup \Gamma_{2}\right)\end{cases}
$$

Let us remark that this problem means that, if $V=\left\{v \in H^{1}(\Omega): v=0\right.$ on $\left.\Gamma_{1} \cup \Gamma_{3}\right\}$, then $u \in V$ satisfies

$$
\int_{\Omega} \nabla u \cdot \nabla v \mathrm{~d} x=\int_{\Omega} f v \mathrm{~d} x, \quad \forall v \in V .
$$

Thanks to Grisvard [16, Section 2.4] complemented by the above paragraph, and since the four corners of $\Omega$ are right angles, we know that the unique solution $u$ of (20) (Lax-Milgram Theorem) can be written as $u=w+\varphi$ where $w \in H^{2}(\Omega)$ and $\varphi$ is given by (18).

Recall that $\mathcal{T}_{H}$ denotes a regular triangulation over $\bar{\Omega}$ with triangles $K$. We require that the origin be a node of $\mathcal{T}_{H}$. Let $u_{H} \in V_{H}=\left\{\psi \in C^{0}(\bar{\Omega}):\left.\psi\right|_{K} \in \mathbb{P}_{1}(K), \forall K \in \mathcal{T}_{H}\right.$ and $\psi=0$ on $\left.\Gamma_{1} \cup \Gamma_{3}\right\}$ be the approximation of $u$ such that

$$
\int_{\Omega} \nabla u_{H} \cdot \nabla v_{H} \mathrm{~d} x=\int_{\Omega} f v_{H} \mathrm{~d} x, \quad \forall v_{H} \in V_{H} .
$$

Using Céa's Lemma we have $\int_{\Omega}\left|\nabla\left(u-u_{H}\right)\right|^{2} \mathrm{~d} x=\int_{\Omega} \nabla\left(u-u_{H}\right) \cdot \nabla\left(u-v_{H}\right) \mathrm{d} x$, and consequently $\left|u-u_{H}\right|_{H^{1}(\Omega)} \leq$ $\left|u-v_{H}\right|_{H^{1}(\Omega)}$, where $|\cdot|_{H^{1}(\Omega)}$ is the semi-norm in $H^{1}(\Omega)$. Since the Poincaré inequality is satisfied in $V$, we have by 
taking $v_{H}=r_{H} w+\varphi_{H}$ where $\varphi_{H} \in V_{H}$ is the function constructed in Lemma $1.2,\left|u-u_{H}\right|_{H^{1}(\Omega)} \leq\left|u-r_{H} u\right|_{H^{1}(\Omega)}$. By Lemma 1.2 and standard interpolation results we have the following a priori error estimate:

$$
\left\|u-u_{H}\right\|_{H^{1}(\Omega)} \leq C\left(c_{0} H^{\frac{1}{2}}+H|w|_{H^{2}(\Omega)}\right)
$$

where $C$ is a constant independent of $H$ and $u$. Hence, the a priori convergence order in $H$ in the $H^{1}$-norm is only of order $1 / 2$.

The objective of the next part will be to apply the correction method to the present situation. We use a patch $\Lambda$ with a finer triangulation to augment the precision around the origin where the first derivative of the solution $u$ explodes in $O$. By strategically choosing the patch we try to optimize the convergence order.

\subsection{Improving the a priori convergence order through using patches.}

In this paragraph we keep considering the model problem (19) introduced above with its approximation (21). The aim is to use the correction algorithm to obtain a better a priori convergence order.

We consider two families of regular triangulations $\mathcal{T}_{H}$ over $\bar{\Omega}$ and $\mathcal{T}_{h}$ over a patch $\bar{\Lambda}_{\epsilon}$, the size of which depends on $\epsilon>0$ in the sense that $\Lambda_{\epsilon} \supset C_{\epsilon}$. We suppose that $\bar{\Lambda}_{\epsilon} \cap \Gamma_{3}=\varnothing$, see Figure 3 with $L=l=1$. Then the idea is to use piecewise polynomial functions of degree 1 on $K \in \mathcal{T}_{H}$ resp. $\mathcal{T}_{h}$, and to approximate the solution $u$ of (19) with $u_{H h}=u_{H}+u_{h}, u_{H}$ and $u_{h}$ defined on $\mathcal{T}_{H}$ and $\mathcal{T}_{h}$ by the correction algorithm described in the Introduction. More precisely, we keep the finite element space $V_{H}$ from the preceding section, and we introduce the new one

$$
\left.V_{h}=\left\{\psi \in H^{1}(\Omega):\left.\psi\right|_{K} \in \mathbb{P}_{1}(K), \forall K \in \mathcal{T}_{h} \text { and } \psi=0 \text { in } \Omega \backslash \Lambda_{\epsilon} \text { and } \psi=0 \text { on } \Gamma_{1} \cap \partial \Lambda_{\epsilon}\right)\right\}
$$

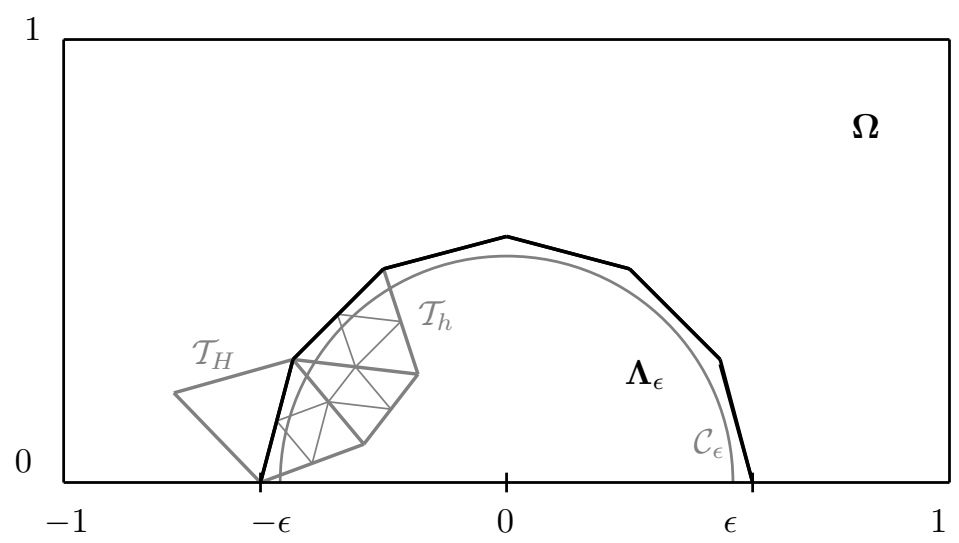

FIGURE 3. Illustration of the setting and notation.

Proposition 2.1. If $u$ is the solution of (19) and if $\mathbf{0}$ is a node of both $\mathcal{T}_{H}$ and $\mathcal{T}_{h}$ then there exists $C$ (independent of $H, h$ and $\epsilon$ ) such that the approximation $u_{H h}$ defined by (2) satisfies the a priori error estimate

$$
\left|u-u_{H h}\right|_{H^{1}(\Omega)} \leq C\left(\frac{H}{\sqrt{\epsilon}}+\sqrt{h}\right) .
$$

Proof. Since $u$ is the solution of (19) we have that $u=w+\varphi$, where $w \in V \cap H^{2}(\Omega)$ and $\varphi$ is given by (18), i.e. $\varphi=c_{0} \sqrt{r} \sin (\theta / 2)$. Hence we obtain by using Céa's Lemma, i.e. $\left|u-u_{H h}\right|_{H^{1}(\Omega)} \leq\left|u-v_{H h}\right|_{H^{1}(\Omega)}, \forall v_{H h} \in V_{H h}$,

$$
\left|u-u_{H h}\right|_{H^{1}(\Omega)} \leq\left|w-r_{H} w\right|_{H^{1}(\Omega)}+\left|\varphi-\varphi_{H h}\right|_{H^{1}(\Omega)},
$$


where $\varphi_{H h}$ is the function constructed in Proposition 1.3 with $\gamma=\frac{1}{2}$ in (4) and $\Gamma=\Gamma_{1}$ in (9) and (11). Note that $\left.r_{H} w\right|_{\Gamma_{1}}=\left.\varphi_{H h}\right|_{\Gamma_{1}}=0$ and $\left.r_{H} w\right|_{\Gamma_{3}}=-r_{H} \varphi_{\Gamma_{3}}=-\left.\varphi_{H h}\right|_{\Gamma_{3}}$, so indeed $r_{H} w+\varphi_{H h} \in V_{H h}$.

Standard interpolation results yield

$$
\left|w-r_{H} w\right|_{H^{1}(\Omega)} \leq C H|w|_{H^{2}(\Omega)}
$$

and we conclude by applying the estimate (12).

It is of interest to write out in Proposition 2.1 the case $\epsilon=\alpha H^{\beta}$, where $\alpha$ and $\beta$ denote constants, $\beta<1$ :

$$
\left|u-u_{H h}\right|_{H^{1}(\Omega)} \sim\left(H^{1-\beta / 2}+h^{1 / 2}\right) .
$$

In the case where $\beta=0$, i.e. the patch is fixed $(\epsilon=\alpha)$, we note that convergence of order one in $H$ can be obtained when choosing $h$ proportional to $H^{2}$. It is adequate to note here that Grisvard proves, under certain conditions [15, Theorem 8.4.1.6] on the local refinement of a family of triangulations, that optimal convergence order, i.e. order one in $H^{1}(\Omega)$-norm, can be reached despite the singularity [15, Corollary 8.4.1.7]. In the present situation of a solution in $W^{2, p}(\Omega)$ with $p \in\left[1 ; \frac{4}{3}\right)$, the conditions of Grisvard's Theorem 8.4.1.6 [15] aim that, as $H \rightarrow 0$, there exists a constant $\sigma$ such that

(i) $\max _{K \in \mathcal{T}_{H}} H_{K} / \rho_{K} \leq \sigma$ where $H_{K}$ is the diameter and $\rho_{K}$ the interior diameter of $K$, i.e., the family of triangulations is regular;

(ii) $H_{K} \leq \sigma H^{2}$, for any triangle $K$ with one corner at the origin;

(iii) $H_{K} \leq \sigma H \inf _{K} r^{1 / 2}$, for any triangle $K$ without corner at the origin.

Condition (ii) of the above result on the refined families requires that the diameter of triangles around the origin is of order $H^{2}$. The above discussion of Proposition 2.1 also implies this crucial condition which can also be found in the work [20] by Raugel.

We remark that if $\epsilon=H,(26)$ becomes $\left|u-u_{H h}\right|_{H^{1}(\Omega)} \sim(\sqrt{H}+\sqrt{h})$, which leads to an estimate $O(\sqrt{H})$ when $h=H$, showing that such a patch is useless.

As we will see in the sequel, it is of practical interest to choose patches of variable size when refining, in particular with respect to memory usage and computation time.

\subsection{Numerical results.}

In order to assess the given error estimate, we consider the problem of approximating $u=w+\varphi$ with $w=0$ and $\varphi$ given by (18). We consider the geometry illustrated in Figure 2. Numerical tests when solving the problem of finding $u \in H^{1}(\Omega)$ such that

$$
\begin{cases}-\Delta u=0 & \text { in } \Omega, \\ u=0 & \text { on } \Gamma_{1}, \\ \frac{\partial u}{\partial n}=0 & \text { on } \Gamma_{2}, \\ u=\left.\varphi\right|_{\Gamma_{3}} & \text { on } \Gamma_{3},\end{cases}
$$

yielding obviously $u=\varphi$, are reported in the following. An illustration of the isolines of the solution $u=\varphi$ is given in Figure 4.

Over $\bar{\Omega}=[-L ; L] \times[0 ; l]$ with $L=l=1$ we consider an unstructured regular triangulation $\mathcal{T}_{H}$ using a discretization of $(-L ; L)$ with $N$ intervals and $(0 ; l)$ with $N / 2$ intervals. We choose $\Lambda_{\epsilon}=(-\epsilon ; \epsilon) \times(0 ; \epsilon)$ and consider $\mathcal{T}_{h}$ a regular and unstructured triangulation based on a discretization of $(-\epsilon ; \epsilon)$ with $M$ intervals and $(0 ; \epsilon)$ with $M / 2$ intervals. In Figure 5 we illustrate the grid constellation with $\epsilon=0.25$ and $N=M=8$.

Accordingly with the discussion at the end of the last paragraph, we choose $\epsilon=\alpha H^{\beta}, \beta<1$, and $h=H^{2-\beta}$ so that the two terms in (26) are of the same order in $H$. With the above notation this is obtained when choosing $M=\alpha^{2} 2^{2 \beta-1} N^{2-2 \beta}$. (If this formula yields a non integer number, we take its integer part.) By Proposition 2.1 we expect that a priori order of convergence in $H$ for the $H^{1}(\Omega)$-norm error is given by $1-\beta / 2$. An overview 


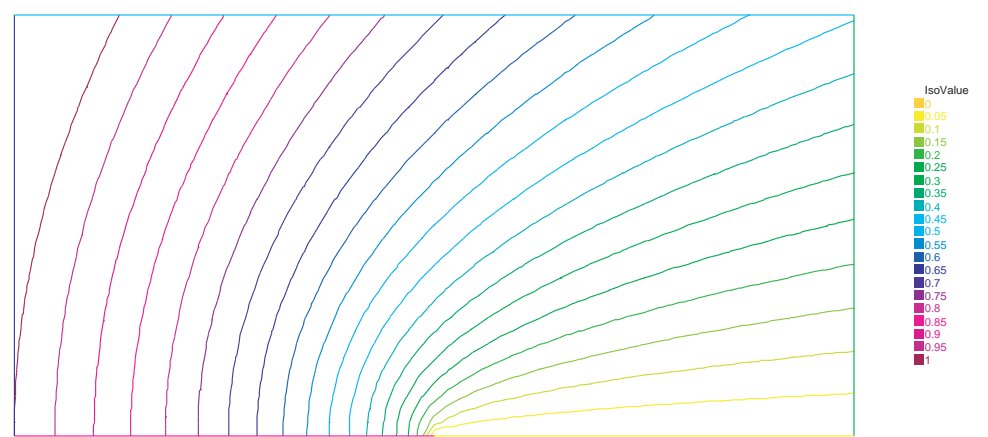

FigURE 4. Isolines of the solution $u=\varphi$ of problem (27).

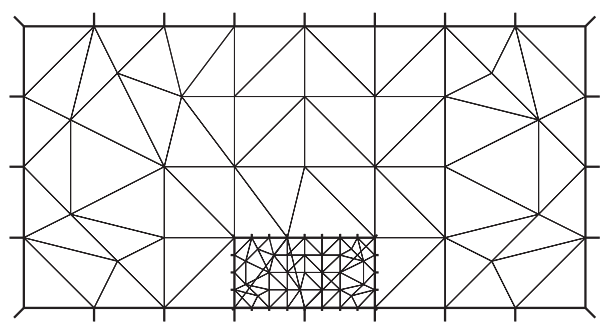

FiguRE 5. Grid constellation showing a patch with $\epsilon=0.25$ and $N=M=8$.

\begin{tabular}{ccccccc}
\hline$\alpha$ & $\beta$ & $\epsilon$ & $h$ & $M$ & CO1 & CO2 \\
\hline 0.25 & 0 & 0.25 & $H^{2}$ & $N^{2} / 32$ & 1 & 1.06 \\
1 & $1 / 4$ & $H^{1 / 4}$ & $H^{7 / 4}$ & $N^{3 / 2} / \sqrt{2}$ & $7 / 8=0.875$ & 0.85 \\
1 & $1 / 2$ & $\sqrt{H}$ & $H^{3 / 2}$ & $N$ & $3 / 4=0.750$ & 0.72 \\
1 & $3 / 4$ & $H^{3 / 4}$ & $H^{5 / 4}$ & $N^{1 / 2} \sqrt{2}$ & $5 / 8=0.625$ & 0.61 \\
\hline \multicolumn{7}{c}{ no patch } \\
\hline
\end{tabular}

TABLE 1. Synoptic table of chosen patches and $H^{1}(\Omega)$-norm convergence orders (CO1 $=$ a priori order in $H, \mathrm{CO} 2=$ order obtained by numerical experience).

of different cases of patch size in function of $H$ applied on the solution of problem (27) is reported in Table 1. In the last column of the latter we report the numerically obtained order in $H$. This value corresponds to the slope (in logarithmic scale) at the last level of refinement of the decreasing relative $H^{1}(\Omega)$-norm error in the mesh size $H$. This is readily seen from Figure 6(a) where we illustrate the concluding convergence behavior in the mesh-size graphically.

Note that the option with $\epsilon=0.25$ makes the number of nodes of the problem of the correction level grow like $M^{2}$ proportional to $N^{4}$ as $H$ decreases. In Figure 6(b) we assess the error reduction with respect to the number of discretization points used. With comparison to solving the problem without patch, the method using a fixed patch with number of discretization points increasing as $N^{4}$ for decreasing $H$ is memory consuming and not satisfactory. A fixed patch is uninteresting in terms of memory usage. However the correction method using a variable patch is economic with respect to memory usage. Well applied patches decrease the error efficiently. In particular when high precision is needed the variable patch is most interesting. 


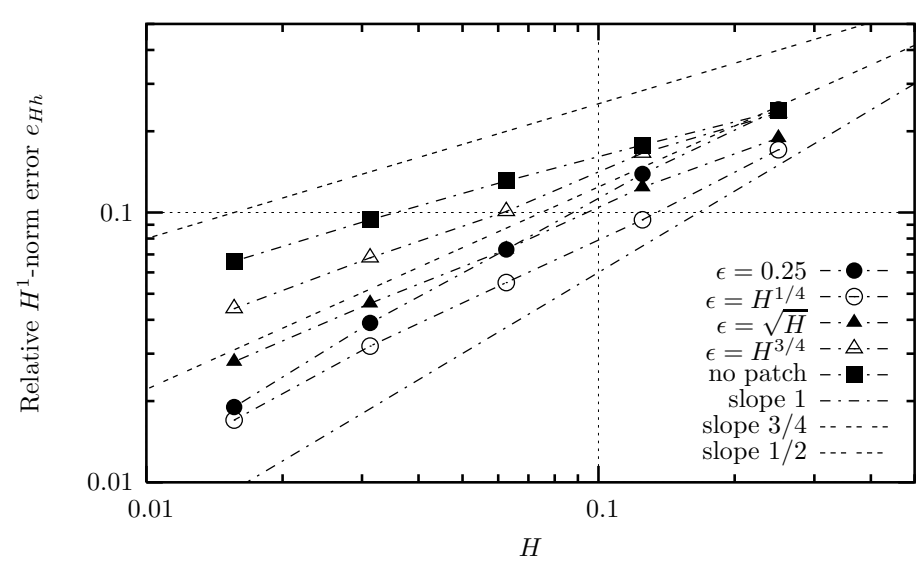

(a) Convergence order in the mesh size.

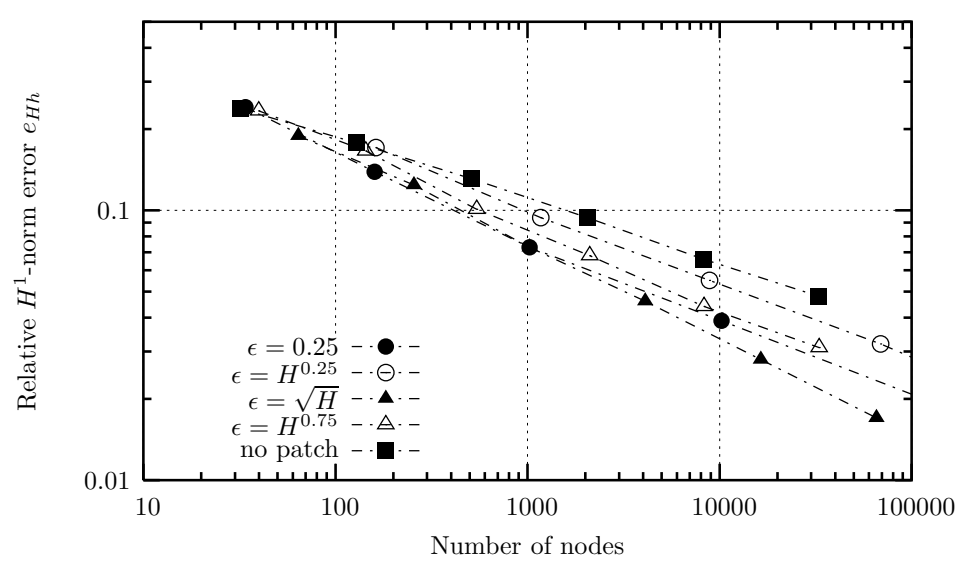

(b) Error reduction vs. number of nodes.

Figure 6 . Convergence of $u_{H}$ resp. $u_{H h}$ to $u$ with respect to the mesh size $H$ and the number of nodes.

The results of this model problem with a singularity due to the change in the boundary conditions are transferable to singularities whose origin lies in a computational domain with reentrant corner. This study is presented in the next section.

\section{Problem in A DOMAin With REENTRANT CORNER}

In the previous section we have discussed the Poisson problem in a rectangular domain with changing Dirichlet-Neumann boundary conditions. In the sequel, the objective is to examine a Poisson-Dirichlet problem in domains with reentrant corners. This will be done similarly to the foregoing analysis.

\subsection{Regularity result.}

A brief analysis of this situation can be found in Grisvard [15, Sections 4.4 and 8.4] and [16, Pages 49-51 and Section 2.4]. It is useful to recall the results here.

Consider the domain $\Omega_{\infty} \subset \mathbb{R}^{2}$ as depicted in Figure 7. We consider for $\Omega_{\infty}$ the part of $\mathbb{R}^{2}$ where the internal angle $\eta$ at the origin on the boundary $\Gamma_{\infty}$ is such that $\pi<\eta<2 \pi$. We consider the problem of finding the 
functions $v \in H_{\mathrm{loc}}^{1}\left(\bar{\Omega}_{\infty}\right)$ verifying $\Delta v=0$ in $\Omega_{\infty}$ with homogeneous Dirichlet boundary conditions on $\Gamma_{\infty}$. We consider polar coordinates centered at the corner $O$ of $\Gamma_{\infty}$. The situation and the notation are illustrated in Figure 7.

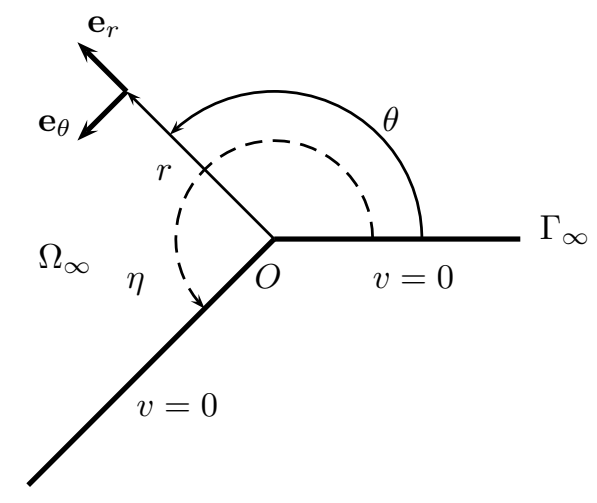

FIGURE 7. Illustration of the situation and notation.

We follow the same analysis of the solution as in the previous section. Taking into account the shape of the domain and the boundary conditions, we consider the function $v=v(r, \theta)$ of the form

$$
v(r, \theta)=\sum_{m \geq 1} \rho_{m}(r) \sin \left(m \frac{\pi}{\eta} \theta\right) .
$$

With the same techniques we show that $\rho_{m}(r)$ is of the form $c_{m} r^{m \pi / \eta}$ for obtaining $v \in H_{\text {loc }}^{1}\left(\bar{\Omega}_{\infty}\right)$.

Considering the second derivatives of $v$, we note that, if $c_{1} \neq 0$, then $v$ does not belong to $H_{\text {loc }}^{2}\left(\bar{\Omega}_{\infty}\right)$. Thus we are interested in calculating $p$ such that $v$ with $c_{1} \neq 0$ is in $W_{\text {loc }}^{2, p}\left(\bar{\Omega}_{\infty}\right)$. For finding such $p$, we evaluate the second derivative of $r^{\pi / \eta}\left(c_{1} \neq 0\right)$ and require it to be $p$-integrable. We obtain the relation $\left(\frac{\pi}{\eta}-2\right) p+1>-1$, and hence $p<\frac{2}{2-\pi / \eta}$.

In conclusion, if $v \in H_{\text {loc }}^{1}\left(\bar{\Omega}_{\infty}\right)$ is an harmonic function in $\Omega_{\infty}$ verifying the homogeneous Dirichlet boundary condition on $\Gamma_{\infty}$, then $v \in W_{\text {loc }}^{2, p}\left(\bar{\Omega}_{\infty}\right)$ with $p \in\left[1 ; \frac{2}{2-\pi / \eta}\right)$. Note that in the extremal case where $\eta \rightarrow 2 \pi$ we are left with $W_{\text {loc }}^{2, p}$-regularity, $p \in\left[1 ; \frac{4}{3}\right)$. Thus the regularity of this extreme situation in the current problem of a domain with reentrant corner corresponds to the regularity of the problem with changing Dirichlet-Neumann boundary conditions on a straight boundary as studied in Section 2.

\subsection{Model problem and a priori error estimate.}

Let the domain $\Omega \subset(-L ; L)^{2} \subset \mathbb{R}^{2}$ be the L-shaped domain as depicted in Figure 8 . We consider the following Poisson problem with homogeneous Dirichlet boundary conditions:

For given $f \in L^{2}(\Omega)$, find $u \in H^{1}(\Omega)$ such that

$$
\begin{cases}-\Delta u=f & \text { in } \Omega, \\ u=0 & \text { on } \partial \Omega .\end{cases}
$$

Thanks to Grisvard [16, Section 2.4] complemented by the above paragraph with $\eta=3 \pi / 2$, we know that the unique solution $u$ of $(29)$ can be written as $u=w+\varphi$ where $w \in H^{2}(\Omega)$ and

$$
\varphi(r, \theta)=c_{1} r^{2 / 3} \sin (2 \theta / 3), .
$$




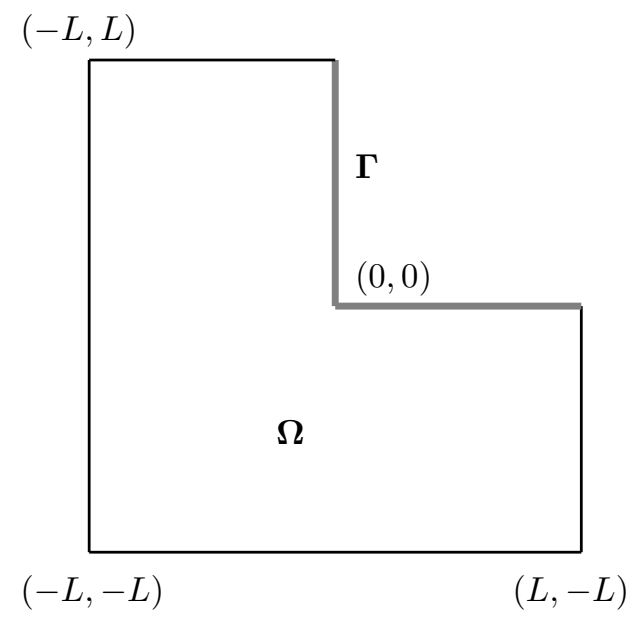

FIGURE 8. Illustration of the domain $\Omega$.

Recall that $\mathcal{T}_{H}$ denotes a regular triangulation over $\bar{\Omega}$ with triangles $K$. We call $H=\max _{K \in \mathcal{T}_{H}} \operatorname{diam}(K)$. By Proposition 1.2 we have the following a priori error estimate:

$$
\left|u-u_{H}\right|_{H^{1}(\Omega)} \leq C\left(c_{0} H^{\frac{2}{3}}+H|w|_{H^{2}(\Omega)}\right),
$$

where $C$ is a constant independent of $H$ and $u$. Hence, the a priori convergence order in $H$ in the $H^{1}$-norm is only $2 / 3$.

\subsection{Improving the a priori convergence order through using patches.}

In this section we keep considering the model problem (29) introduced above. The aim is to use the correction algorithm to obtain a better a priori convergence order. Complete analysis alike in Section 2 can be performed and leads to a particular result similar to Proposition 2.1 adapted to the current problem. We will not reiterate such a reasoning here.

Consider two families of regular triangulations $\mathcal{T}_{H}$ over $\bar{\Omega}$ (as above) and $\mathcal{T}_{h}$ over $\bar{\Lambda}_{\epsilon}, \Lambda_{\epsilon}=(-\epsilon ; \epsilon)^{2} \cap \Omega$. Recall that $H=\max _{K \in \mathcal{T}_{H}} \operatorname{diam}(K)$ and call $h=\max _{K \in \mathcal{T}_{h}} \operatorname{diam}(K)$ the diameter of the triangles $K$. We use hat function elements and approximate the solution $u$ of (29) with $u_{H h}=u_{H}+u_{h}, u_{H}$ and $u_{h}$ defined by using $\mathcal{T}_{H}$ and $\mathcal{T}_{h}$ respectively and by using the correction method given in the Introduction.

We are able to prove in the same way as Proposition 2.1 that there exists $C$ such that the approximation $u_{H h}$ to $u$ satisfies the a priori error estimate

$$
\left|u-u_{H h}\right|_{H^{1}(\Omega)} \leq C\left(\frac{H}{\epsilon^{1 / 3}}+h^{2 / 3}\right) .
$$

It is of interest to write out (32) when $\epsilon=\alpha H^{\beta}$, where $\alpha$ and $\beta$ denote constants, $\beta<1$ :

$$
\left|u-u_{H h}\right|_{H^{1}(\Omega)} \sim\left(H^{1-\beta / 3}+h^{2 / 3}\right) .
$$

Hence when $\epsilon$ is proportional to $H^{\beta}$, we choose $h$ proportional to $H^{3 / 2-\beta / 2}$ to optimize the estimate and obtain a convergence of order $1-\beta / 3$ in $H$. 


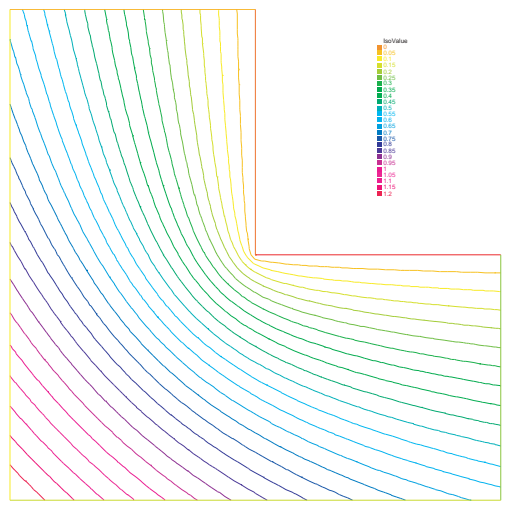

FigURE 9. Isolines of the solution $u=\varphi$ of problem (34).

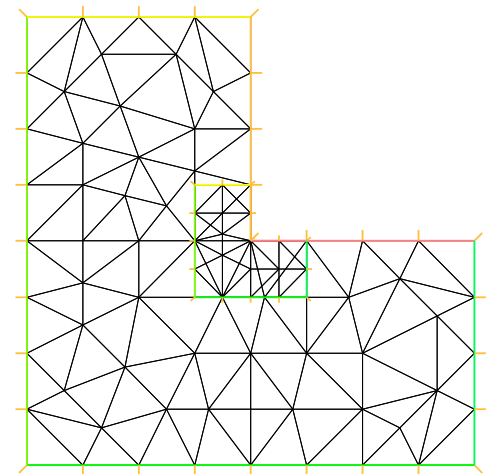

FiguRE 10. Illustration of the used grid constellation with $\epsilon=0.25$ and $N=8, M=4$.

\subsection{Numerical results.}

In order to assess the given error estimates, we consider the problem of approximating $u=w+\varphi$ with $w=0$ and $\varphi$ given by 30 . Numerical tests when solving the problem of finding $u \in H^{1}(\Omega)$ such that

$$
\begin{cases}-\Delta u=0 & \text { in } \Omega \\ u=\left.\varphi\right|_{\partial \Omega} & \text { on } \partial \Omega\end{cases}
$$

yielding obviously $u=\varphi$, are reported in the following. An illustration of the isolines of the solution $u=\varphi$ is given in Figure 9.

We recall the triangulations $\mathcal{T}_{H}$ over $\bar{\Omega}$ and $\mathcal{T}_{h}$ over $\bar{\Lambda}_{\epsilon}$. We consider a discretization of $(-L ; L)$ with $N$ intervals and set $L=1$. For $(-\epsilon ; \epsilon)$ we use $M$ intervals. In Figure 10 we illustrate the situation by the triangulations used for $\epsilon=0.25$ and $N=8, M=4$.

Accordingly to the above discussion, we choose $\epsilon=\alpha H^{\beta}, \beta<1$, and accordingly $h=H^{3 / 2-\beta / 2}$. With the above notation this is obtained when choosing $M=\alpha^{3 / 2} 2^{3 \beta / 2-1 / 2} N^{3 / 2-3 \beta / 2}$. The a priori order of convergence in $H$ for the $H^{1}(\Omega)$-norm error is given by $1-\beta / 3$. An overview of different cases applied on the solution of problem (34) is reported in Table 2. In the last column of the latter we report the numerically obtained order in $H$. In Figure 6(a) we illustrate the concluding convergence behavior in the mesh-size graphically.

In Figure 6(b) we assess the error reduction with respect to the total number of nodes used. 


\begin{tabular}{ccccccc}
\hline$\alpha$ & $\beta$ & $\epsilon$ & $h$ & $M$ & CO1 & CO2 \\
\hline 0.25 & 0 & 0.25 & $H^{3 / 2}$ & $N^{3 / 2} / 2^{7 / 2}$ & 1 & 0.93 \\
1 & $1 / 3$ & $H^{1 / 3}$ & $H^{4 / 3}$ & $N$ & $8 / 9 \approx 0.89$ & 0.79 \\
1 & $2 / 3$ & $H^{2 / 3}$ & $H^{7 / 6}$ & $N^{1 / 2} \sqrt{2}$ & $7 / 9 \approx 0.78$ & 0.74 \\
\hline \multicolumn{7}{c}{ no patch } \\
\end{tabular}

TABLE 2. Synoptic table of chosen patches and $H^{1}(\Omega)$-norm convergence orders $(\mathrm{CO} 1=a$ priori order in $H, \mathrm{CO} 2=$ order obtained by numerical experience).

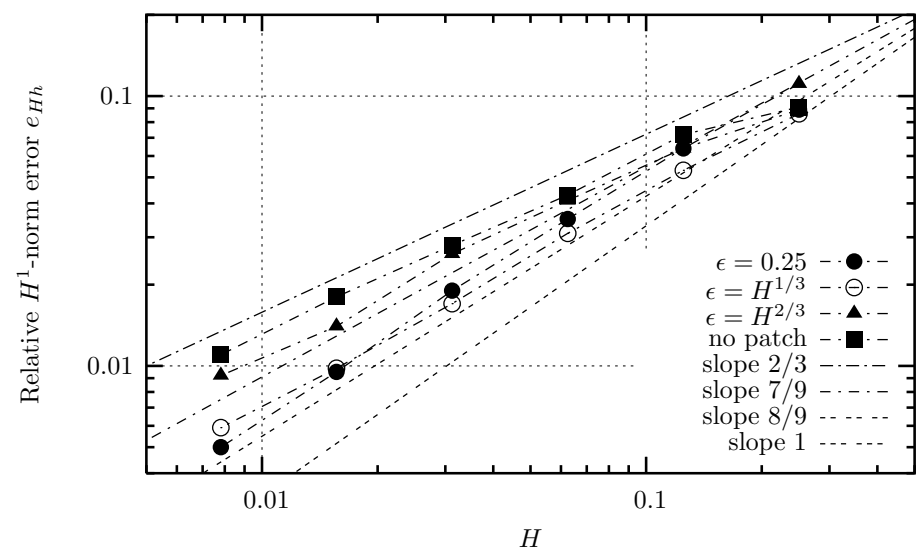

(a) Convergence order in the mesh size.

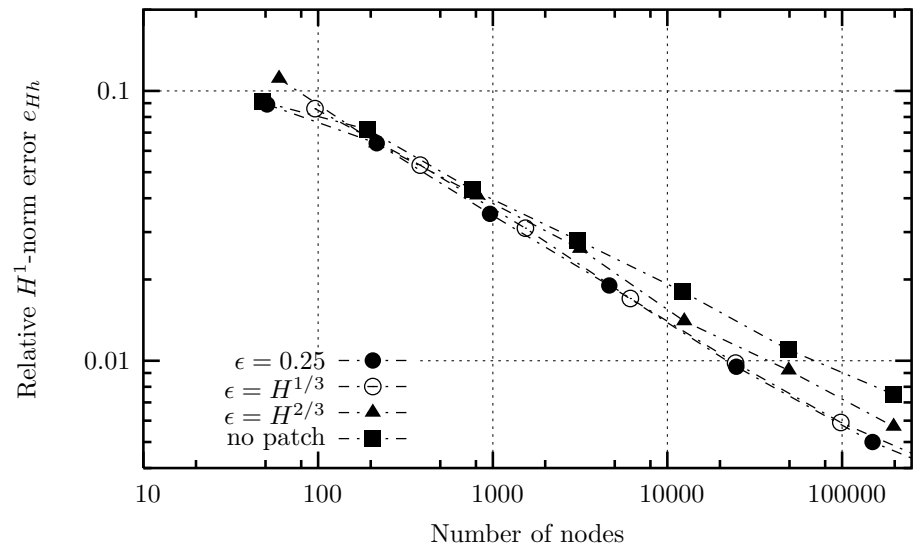

(b) Error reduction vs. number of nodes.

Figure 11. Convergence of $u_{H}$ resp. $u_{H h}$ to $u$ with respect to the mesh size $H$ and the number of nodes.

\section{Applichtion to glacier modeling}

The objective of this section is to apply the correction method to the modeling of glaciers. Ice is usually treated as incompressible viscous (in the case of cold ice) heat conducting fluid, with a Glen-type rheology [10]. 
The basic continuum mechanics equation for mass conservation, momentum conservation and constitutive relation for such an ice flow have been studied by Fowler and Larson [9], Hutter [18] and Morland [19]. We are going to use in this work a model by Blatter [2], and for numerical studies consider a 2D vertical cut of the Gries glacier (Switzerland) in the direction of the motion of a glacier and horizontal invariance (the glacier is very large), as studied by Reist [21]. The model we use allows to simulate the horizontal velocity field of the ice mass and the effective stress. Changes in the basal surface, the interface between the ice mass and the rock bed, imply different conditions for the flow. We suppose that boundary conditions can change from free sliding to prescribed velocity, and reverse, along the basis. This directly relates to the solution for the velocity and thus for the stress field which is more or less regular. A good precision is necessary as the change from Neumann to Dirichlet conditions reflects non-locally in variations all over the ice mass.

\subsection{A mathematical model of a glacier.}

The geometry of the 2D vertical cut of the ice mass is defined by the upper free surface $S$ given by $z=S(x)$, $x \in[a ; b]$, which is supposed regular (i.e. no overhanging), and the basal surface $B$ given by $z=B(x), x \in[a ; b]$, in cartesian coordinates $(x, z)$ with the $z$-axis pointing opposite the direction of gravity. We call $\Gamma_{S}$ the upper surface of the glacier given by the points $(x, z)$ such that $z=S(x)$ and $\Gamma_{B}$ the mountain base, the points $(x, z)$ such that $z=B(x)$. We suppose that $B(x) \leq S(x)$ and denote by $\Omega$ the glacier domain occupied by ice, the set of points $(x, z)$ such that $B(x) \leq z \leq S(x), x \in[a ; b]$. Furthermore we split $\Gamma_{B}$ in two parts $\Gamma_{B, D}$ where we consider the glacier fixed on the mountain, i.e. homogeneous Dirichlet boundary conditions for the velocity field, and $\Gamma_{B, N}$ where we consider Neumann boundary conditions for the velocity which represent a slip condition.

The model by Blatter [2] rewritten by Wagner in [22, Chapter 4] leads to the following problem: Find the horizontal velocity $u$ defined in $\Omega$ such that

$$
\begin{cases}-\operatorname{div}(\mu(|\nabla u|) \nabla u)=f & \text { in } \Omega \\ \frac{\partial u}{\partial n}=0 & \text { on } \Gamma_{S}, \\ \frac{\partial u}{\partial n}=0 & \text { on } \Gamma_{B, N} \\ u=0 & \text { on } \Gamma_{B, D}\end{cases}
$$

where $f=-\frac{1}{4} \rho g \frac{\partial S}{\partial x}, \rho$ being the density of ice and $g$ the acceleration of gravity, and $\mu=\mu(s)$ is given by

$$
A\left([\mu(s)]^{n-1} s^{n-1}+\sigma_{0}^{n-1}\right)=\frac{1}{2 \mu(s)},
$$

with $A$ and $\sigma_{0}$ constants, and $n \geq 1$ an exponent. In [13, Lemma 1] it is proven that $\mu$ as a function of $s$ is well defined by relation (36). We observe that for $n=1, \mu(s)=1 / 4 A$, and for $n=2, \mu$ is readily explicited, $\mu(s)=\left(-A \sigma_{0}+\sqrt{A^{2} \sigma_{0}^{2}+2 A s}\right) / 2 A s$.

The horizontal velocity field $u$ is given by the above problem (35). Furthermore the effective stress field $\sigma_{(I I)}$ is expressed through

$$
\sigma_{(I I)}=\mu(|\nabla u|)|\nabla u| \text {. }
$$

We will compute the velocity field of the glacier by solving numerically problem (35). We will use a finite element method, using a computational mesh adapted to the geometry of the problem.

\subsection{Weak formulation.}

We establish a weak formulation of problem (35). By multiplying the first equation of the latter system by $\varphi$ vanishing on $\Gamma_{B, D}$, by integrating by part on $\Omega$ and taking into account the natural boundary conditions (second and third equation of $(35))$ on $\Gamma_{S}$ and $\Gamma_{B, N}$, by setting

$$
a_{\mu}(u, \varphi)=\int_{\Omega} \mu(|\nabla u|) \nabla u \cdot \nabla \varphi \mathrm{d} \Omega
$$


we obtain

$$
a_{\mu}(u, \varphi)=\int_{\Omega} f \varphi \mathrm{d} \Omega
$$

Remark that $a_{\mu}(u, \varphi)$ is linear with respect to $\varphi$, but nonlinear with respect to $u$ because $\mu$ depends on $u$. Let now $V$ be the Banach space, $V=\left\{\varphi \in W^{1, p}(\Omega): \varphi=0\right.$ on $\left.\Gamma_{B, D}\right\}$, where $p=\frac{n+1}{n}$, with $n$ the exponent appearing in the flow law (36). In [13] it is proven that there exist $c_{1}, c_{2}>0$ such that

$$
c_{1}(1+|\nabla u|)^{\frac{1}{n}-1} \leq \mu(|\nabla u|) \leq c_{2}(1+|\nabla u|)^{\frac{1}{n}-1}, \quad \forall|\nabla u| \in(0 ;+\infty),
$$

and hence $\mu(|\nabla u|) \nabla u \in L^{q}(\Omega)$ with $\frac{1}{p}+\frac{1}{q}=1(q=n+1)$, when $u \in V$. Consequently, the form (38) makes sense when $u, \varphi \in V$.

It follows that the weak formulation of problem (35) with natural boundary conditions on the upper surface $\Gamma_{S}$ and the part $\Gamma_{B, N}$ of the basal surface, and Dirichlet conditions on the part $\Gamma_{B, D}$ of the basal surface, is:

Find $u \in V$ such that

$$
a_{\mu}(u, \varphi)=\int_{\Omega} f \varphi \mathrm{d} \Omega, \quad \forall \varphi \in V .
$$

Colinge and Rappaz prove in [7, Theorem 1] the uniqueness of the solution in the case of Dirichlet boundary conditions on all $\partial \Omega$. This result can be extended to our case of problem (41) with mixed Dirichlet-Neumann conditions.

\subsection{Discretization.}

We discretize problem (41) by using hat functions. First we choose the mesh size $H$ and consider an approximation of $\Omega$ by a polygonal domain which is still denoted by $\Omega$ with sides of length $H$. We denote by $\mathcal{T}_{H}$ a regular triangulation of $\bar{\Omega}$ with triangles $K \in \mathcal{T}_{H}$ such that $\forall K \in \mathcal{T}_{H}, \operatorname{diam}(K) \leq H$. We assume that for each triangle $K \in \mathcal{T}_{H}, K \cap \Gamma_{B, N}$ is either void or a side of $K$ or a vertex of $K$, where $\Gamma_{B, N}$ denotes the polygonal line of $\partial \Omega$ joining the fixed points $A$ and $B$ (see Figure 12). We introduce $V_{H}=\left\{\psi \in \mathcal{C}^{0}(\bar{\Omega}):\left.\psi\right|_{K} \in\right.$ $\mathbb{P}_{1}(K), \forall K \in \mathcal{T}_{H}$ and $\psi=0$ on $\left.\Gamma_{B, D}\right\}$, where $\Gamma_{B, D}$ denotes the part of $\partial \Omega$ corresponding to the part of the basal surface with Dirichlet boundary conditions, and call $\mu_{H}=\mu\left(\left|\nabla u_{H}\right|\right)$. We consider the following discrete problem: Find $u_{H} \in V_{H}$ such that

$$
a_{\mu_{H}}\left(u_{H}, \varphi_{H}\right)=\int_{\Omega} f \varphi_{H} \mathrm{~d} \Omega, \quad \forall \varphi_{H} \in V_{H}
$$

\subsection{Linearization.}

In order to solve numerically the discrete variational problem (42) we use Picard's iterative method. We apply the following steps:

- Initialization: Set $u_{H, 0}$ the solution of (42) with $n=1$, i.e. $\mu=\mu_{H}=1 / 4 A$.

- For $k=1,2,3, \ldots$ solve the following linear problem: Find $u_{H, k} \in V_{H}$ satisfying

$$
a_{\mu_{H, k-1}}\left(u_{H, k}, \varphi_{H}\right)=\int_{\Omega} f \varphi_{H} \mathrm{~d} \Omega, \quad \forall \varphi_{H} \in V_{H},
$$

where $\mu_{H, k-1}=\mu\left(\left|\nabla u_{H, k-1}\right|\right)$.

A proof of convergence of this algorithm is given by Reist in [21, §2.1.1]. Hence $u_{H, k} \rightarrow u_{H}$ for $k \rightarrow \infty$ [21, Theorem 2.1.3]. 


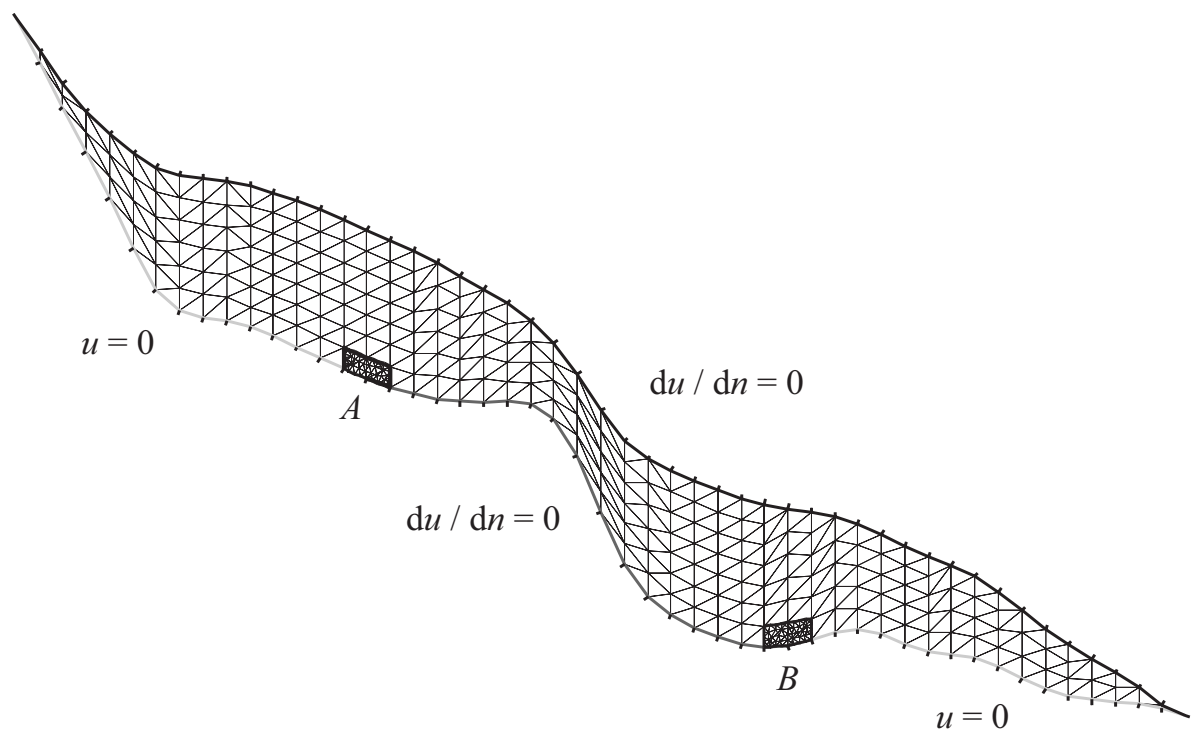

FiguRE 12. Geometry, boundary conditions and triangulation $(N=50)$ of the Gries glacier. Grid constellation and illustration of two patches $(M=4, H / h=4)$ around the points $A$ and $B$.

\subsection{Numerical illustration.}

We illustrate our numerical model for a two-dimensional glacier on a vertical section along a flow line passing through the center of Gries glacier (Wallis, Swiss Alps). As the Gries glacier is large in the transverse direction our two-dimensional computations are relevant. The shape of the glacier [21] and the basic meshing with $N=50$ intervals in the $x$-direction (and accordingly discretized in the $z$-direction to obtain a regular triangulation) is illustrated in Figure 12.

We study the nonlinear case with $n=2$. For defining the right-hand side $f$, defined in (4.1), we set $\rho g=900 \cdot 9.81 \cdot 10^{-5}$ and in the flow law (36) we take $A=0.08$ and $\sigma_{0}=0.1$, as suggested by [21] and converted from the S.I. unit values from Greve [14, p. 936].

We use the relative $L^{2}$-discrepancy of the stress field as stopping criteria for the iterations due to linearization. When running the algorithm described in the Introduction (with a discrepancy of $10^{-3}$ in the corrections), we note that about 10 iterations are required to obtain convergence. We have implemented the model with the software Freefem++ [17].

\subsection{Stress field on the mountain base.}

Let us now turn to the stress field of the glacier and consider the nonlinear case. Before studying how to increase the precision on the approximation of the latter, we briefly outline its behavior along the mountain base, i.e. the boundary $\Gamma_{B}$. Our knowledge of the behavior of the velocity field in the case of the (linear) Poisson problem with changing Dirichlet-Neumann boundary conditions (Section 2), and the result (40) meaning that $\mu$ behaves like $|\nabla u|^{\frac{1}{n}-1}$ when $|\nabla u| \rightarrow \infty$, leads us to conjecture that the effective stress field $\sigma_{(I I)}$ blows up at the points $A$ and $B$. This is confirmed by the illustration in Figure 13 where the values of $\sigma_{(I I)}$ are plotted along $\Gamma_{B}$ in the case $n=2$. A good precision is needed around $A$ and $B$ where the stress field blows up. In the next section, we consider applying patches in these small regions. 


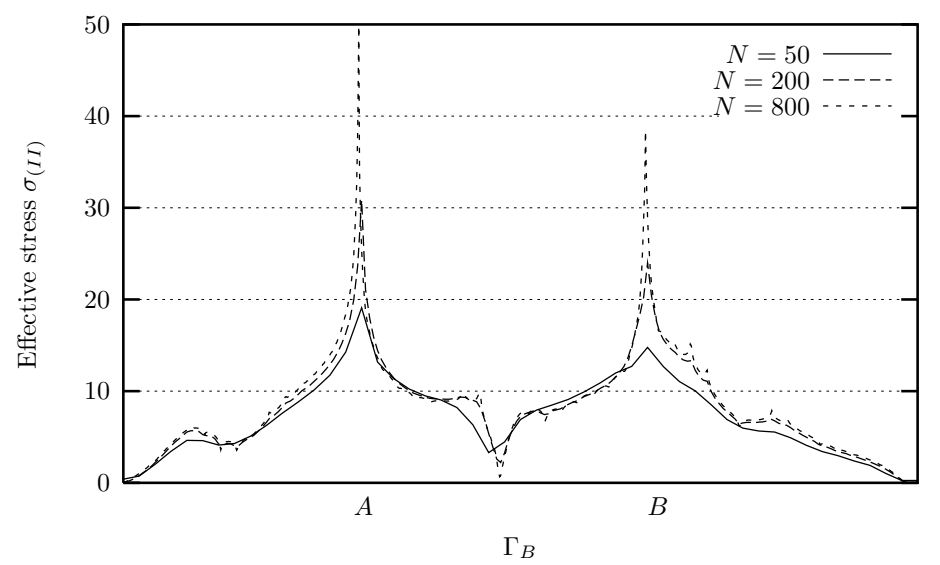

Figure 13. Behavior of the stress field $\sigma_{(I I)}\left(u_{H}\right)$ on $\Gamma_{B}$ in the nonlinear case $n=2$. Numerical values are not scaled to physical units. The Figure merely illustrates the relative behavior for different $N$.

\subsection{Patches and precision of the glacier stress field}

We consider in this section the stress field of the glacier and consider the application of patches. We show that the application of patches is efficient for reducing the error around the points of changing boundary conditions on the basal surface.

The study of the effective stress field (Figure 13) shows that the stress field blows up around the points of the boundary where the boundary conditions change.

With $(37), \sigma_{(I I)}=\mu(|\nabla u|)|\nabla u|$, and (40), the minimal regularity requirement on the velocity field $u \in V$ a subset of $W^{1, p}(\Omega)$ with $p=(n+1) / n$, suffices to have $\sigma_{(I I)} \in L^{1+n}(\Omega)$, i.e. at least $L^{2}(\Omega)$, since $n \geq 1$. Hence the stress field is $L^{2}$-integrable, and the $L^{2}$-norm is adapted to measure it.

In our model, the boundary of the glacier domain $\Omega$ has two points $A$ and $B$ on the basal surface where it presents a Dirichlet-Neumann change in the boundary conditions (see Figure 12). Furthermore, we apply different patches with $(M+1)(M / 2+1)$ discretization points in the regions around $A$ and $B$ in order to see how to sharpen the results.

We consider two types of patches as in Section 2. A first type, called fixed patch $\Lambda_{A}, \Lambda_{B}$, which in the original mesh with $N=50$ points covers exactly one triangle in all directions around $A$ and $B$, and a second type which covers, at any refinement level, one triangle in all directions: it is what we call a variable patch. An illustration of fixed patches and their size for the refined mesh with $N=50$ and $M=4$, i.e. $H / h=4$, is depicted in Figure 12. For the triangulations of $\Lambda_{A}$ and $\Lambda_{B}$, we consider refinements such that the diameter $h$ of the fine triangulation is $h=H / 2, H / 4$ or $H / 8$.

As mentioned previously, about 10 iterations are necessary to cope with the iterative method for the linearized problem. Currently, we have an inside loop corresponding to the iterations of the correction algorithm. Requiring the relative discrepancy of the velocity field in the $L^{2}$-norm to be below $10^{-2}$, the inner-loop convergence needs 2 to 3 full iterations.

We measure the $L^{2}$-norm relative error $(n=2)$ of the stress field $\sigma_{H h}=\sigma_{(I I)}\left(u_{H h}\right)$ with respect to the "almost exact" solution $u$ given by $u_{H}$ with $N=3200$ and $\sigma=\sigma_{(I I)}(u)$. We evaluate the norms in domains $\Lambda_{A}$ and $\Lambda_{B}$ for $N=50$ and 100, and for situations without patch and with patch. For the situation where we apply a patch we consider either two fixed patches or two variable patches (smaller for $N=100$ ) around $A$ and $B$. In each case, the error is evaluated in the region covered by the patch. The respective values are reported in Table 3. 


\begin{tabular}{ccc|cc}
\hline$N$ & $M$ & & $\frac{\left\|\sigma-\sigma_{H h}\right\|_{L^{2}\left(\Lambda_{A}\right)}}{\|\sigma\|_{L^{2}\left(\Lambda_{A}\right)}}$ & $\frac{\left\|\sigma-\sigma_{H h}\right\|_{L^{2}\left(\Lambda_{B}\right)}}{\|\sigma\|_{L^{2}\left(\Lambda_{B}\right)}}$ \\
\hline 50 & - & no patch & 0.264 & 0.310 \\
& 2 & $H / h=2$ & 0.194 & 0.237 \\
4 & $H / h=4$ & 0.168 & 0.170 \\
8 & $H / h=8$ & 0.184 & 0.155 \\
\hline Fixed patch, large region \\
$100 \quad-\quad$ no patch & 0.201 & 0.215 \\
4 & $H / h=2$ & 0.171 & 0.134 \\
8 & $H / h=4$ & 0.181 & 0.120 \\
16 & $H / h=8$ & 0.164 & 0.111 \\
\hline Variable & patch, small region & \\
100 & - & no patch & 0.265 & 0.290 \\
& 2 & $H / h=2$ & 0.225 & 0.220 \\
4 & $H / h=4$ & 0.239 & 0.155 \\
8 & $H / h=8$ & 0.232 & 0.193
\end{tabular}

TABLE 3. $L^{2}$-norm relative error of the velocity and the stress fields in the patches (with $n=2$ ).

Considering the data reported in Table 3, we note that the reduction of the error is efficient. If we evaluate the errors in variable regions, though refining the grids by a factor 2, from $N=50$ to 100 with no patch for example, we note no considerable change in the value of the error as the domain for evaluation has in the same time tightened around the point of singular behavior.

We conclude with a precision gain for the stress field due to the application of patches. Taking for example the case $N=50$ with no patch and a patch with $M=8$ around $B$, we report that the error on the stress field is divided by two! Furthermore, we can also compare the convincing pair of situations of $N=50$ with a patch ( $M=4$ or 8 ) and of $N=100$ with no patch. While using much less discretization points, applying a small, well chosen patch yields better results than a global refinement.

Finally, the question of the optimal size of the patch arises. To give an answer to this point, we consider the coarse discretization with $N=50$ and consider the relative $L^{2}(\Omega)$-norm error of the effective stress field with respect to the above introduced "almost exact" solution.

Without any patch, this error yields 0.153. Applying patches around $A$ and $B$ with the size as shown in Figure 12 (i.e. covering one coarse triangle in each direction around the point $A$ or $B$, the patch is large by $2 H$ ) and a ratio $H / h=2$, we reduce the error and get 0.121 .

If we want to further improve the solution, we can either enlarge the regions over which we consider the patches, or refine the chosen patches. Refining the patches such that $H / h=8$, reduces the error to the value 0.110. However applying patches around $A$ and $B$ that are $8 H$ large (four times larger, four times higher), keeping the ratio $H / h=2$ constant, the relative error does not improve: 0.126 . This means that in this particular situation, the size of the patch is accurately chosen small, covering the region where the solution varies most.

In conclusion, note that the correction method and an accurate analysis for the placement of the patches pays out most when we consider evolution problems (e.g. the modeling of glaciers as done by Reist [21]).

\section{ACKNOWLEGEMENTS}

The authors thank Professor Jiwen He for the interesting discussions and his suggestions concerning the subject. They are also grateful to anonymous referee for the remarks that helped them to improve the presentation of the results. 


\section{REFERENCES}

[1] I. Babuska. Finite element method for domains with corners. Computing (Arch. Elektron. Rechnen), 6:264-273, 1970.

[2] H. Blatter. Velocity and Stress Fields in Grounded Glaciers: A Simple Algorithm for Including Deviatoric Stress Gradients. J. Glac., 41(138):333-344, 1995.

[3] M. Bourlard, M. Dauge, and S. Nicaise. Error estimates on the coefficients obtained by the singular function method. Numer. Funct. Anal. Optim., 10(11-12):1077-1113 (1990), 1989.

[4] S.C. Brenner. Multigrid methods for the computation of singular solutions and stress intensity factors i: corner corner singularities. Math. Comp., 68(226):559-583, 1999.

[5] Z. Cai and S. Kim. A finite element method using singular functions for the Poisson equation: corner singularities. SIAM J. Numer. Anal., 39:286-299, 2001.

[6] P.G. Ciarlet. Basic error estimates for elliptic problems. In P.G. Ciarlet and J.L. Lions, editors, Finite Element Methods (Part 1), volume II of Handbook of Numerical Analysis, pages 17-351. North-Holland, 1991.

[7] J. Colinge and J. Rappaz. A Strongly Nonlinear Problem Arising in Glaciology. M2AN, 33(2):395-406, 1999.

[8] M. Dauge. Elliptic boundary value problems on corner domains, volume 1341 of Lecture Notes in Mathematics. Springer, 1988.

[9] A.C. Fowler and D.A. Larson. On the flow of polythermal glaciers. I. Model and preliminary analysis. Proc. R. Soc. London, Ser. A, 363:217-242, 1978.

[10] J. Glen. The creep of polycrystalline ice. Proc. R. Soc. London, Ser. A, 228(1175):519-538, 1955.

[11] R. Glowinski, J. He, A. Lozinski, J. Rappaz, and J. Wagner. Finite element approximation of multi-scale elliptic problems using patches of elements. Numer. Math., 101(4):663-687, 2005.

[12] R. Glowinski, J. He, J. Rappaz, and J. Wagner. Approximation of multi-scale elliptic problems using patches of finite elements. C. R. Acad. Sci. Paris, Ser. I, 337:679-684, 2003.

[13] R. Glowinski and J. Rappaz. Approximation of a Nonlinear Elliptic Problem in a Non-Newtonian Fluid Flow Model in Glaciology. M2AN, 37(1):175-186, 2003.

[14] R. Greve. A continuum-mechanical formulation for shallow polythermal ice sheets. Phil. Trans. R. Soc. Lond., Ser. A, 355:921974, 1997.

[15] P. Grisvard. Elliptic Problems in Nonsmooth Domains, volume 24 of Monographs and Studies in Mathematics. Pitman, 1985.

[16] P. Grisvard. Singularities in Boundary Value Problems, volume 22 of Research Notes in Applied Mathematics. Masson, 1992.

[17] F. Hecht, O. Pironneau, and K. Ohtsuka. Freefem++. http://www.freefem.org.

[18] K. Hutter. Theoretical Glaciology: Material Science of Ice and the Mechanics of Glaciers and Ice Sheets. Mathematical Approaches to Geophysics. D. Reidel Publishing Company / Terra Scientific Publishing Company, 1983.

[19] L.W. Morland. Thermo-mechanical balances of ice sheet flows. Geophys. Astrophys. Fluid Dyn., 29:237-266, 1984.

[20] G. Raugel. Résolution numérique par une méthode d'éléments finis du problème de Dirichlet pour le laplacien dans un polygone. C. R. Acad. Sci. Paris, Ser. A, 286:791-794, 1978.

[21] A. Reist. Mathematical analysis and numerical simulation of the motion of a glacier. PhD thesis, EPFL, Lausanne, Switzerland, 2005.

[22] J. Wagner. Finite Element Methods with Patches and Applications. PhD thesis, EPFL, Lausanne, Switzerland, 2006. 